\title{
Utility of Inflammatory Markers in Detection of Perioperative Morbidity After Laparoscopic Sleeve Gastrectomy, Laparoscopic Roux-en-Y Gastric Bypass, and One-Anastomosis Gastric Bypass-Multicenter Study
}

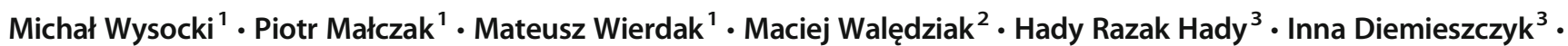 \\ Monika Proczko-Stepaniak ${ }^{4}$ - Michał Szymański ${ }^{4} \cdot$ Natalia Dowgiałło-Wnukiewicz $^{5}$ • Jacek Szeliga ${ }^{6}$. \\ Michał Pędziwiatr ${ }^{1} \cdot$ Piotr Major $^{1}$ (D)
}

Published online: 28 April 2020

(C) The Author(s) 2020

\begin{abstract}
Background The most commonly performed bariatric operations are laparoscopic sleeve gastrectomy (LSG) and bypass surgeries (laparoscopic one-anastomosis gastric bypass (OAGB) and laparoscopic Roux-en-Y gastric bypass (LRYGB)), and predicting perioperative morbidity is crucial for early, safe patient discharge. We aimed to determine whether C-reactive protein (CRP) and white blood count (WBC) measured on the first postoperative day predicts perioperative morbidity in the first 30-days after LSG and bypass surgeries.

Methods We retrospectively analyzed data for 1400 patients who underwent bariatric surgery in seven bariatric centers from 2014 to 2018. Patients were divided into a complicated group (patients with postoperative complications) and a non-complicated group. We also performed separate analyses for LSG and bypass surgeries.

Results Patients were 929 women (66\%) and 471 men (34\%) with a median age of 42 years (range, 35-51 years); 1192 patients underwent LSG (85\%), 120 underwent LRYGB (9\%), and 80 underwent OAGB (6\%). We performed ROC analyses to set cutoff points, followed by multivariate logistic regressions. CRP $>33.32 \mathrm{mg} / \mathrm{L}$ increased the odds ratio (OR) of perioperative complications after LSG 2.27 times, while $\mathrm{WBC}>12.15 \times 10^{3} / \mu \mathrm{L}$ on postoperative day 1 was associated with a 3.34-times greater or of developing complications. $\mathrm{WBC}>13.78 \times 10^{3} / \mu \mathrm{L}$ was associated with a 13.34-times higher or of perioperative morbidity in patients undergoing bypass surgeries.

Conclusion Even slightly elevated CRP and WBC on postoperative day 1 should alert surgeons to the potential risk of perioperative morbidity.
\end{abstract}

Keywords Laparoscopic sleeve gastrectomy · Laparoscopic Roux-en-Y gastric bypass · One-anastomosis gastric bypass · CRP . Inflammatory markers $\cdot$ Morbidity $\cdot$ Mortality

Piotr Major

piotr.major@uj.edu.pl

Michał Wysocki

m.wysocki@doctoral.uj.edu.pl

Piotr Małczak

piotr.malczak@doctoral.uj.edu.pl

Mateusz Wierdak

mateusz.wierdak@uj.edu.pl

Maciej Walędziak

maciej.waledziak@gmail.com

Hady Razak Hady

hadyrazakh@wp.pl

Inna Diemieszczyk

Demeschik.inna@gmail.com

\author{
Monika Proczko-Stepaniak \\ mproczko@gumed.edu.pl \\ Michał Szymański \\ szymanski@gumed.edu.pl \\ Natalia Dowgiałło-Wnukiewicz \\ natalia.dowgiallo@gmail.com \\ Jacek Szeliga \\ jacky2@wp.pl \\ Michał Pędziwiatr \\ michal.pedziwiatr@uj.edu.pl
}

Extended author information available on the last page of the article 


\section{Introduction}

The number of bariatric procedures is increasing annually. According to the 2016 International Federation for the Surgery of Obesity and Metabolic Disorders (IFSO) survey data from 58/62 IFSO Societies, the total number of bariatric/ metabolic procedures performed in 2016 was 685,874 . The most common primary surgical bariatric/metabolic procedure was laparoscopic sleeve gastrectomy (LSG) $(340,550$; $53.6 \%$ ), followed by Roux-en-Y gastric bypass (LRYGB) $(191,326 ; 30.1 \%)$, and one-anastomosis gastric bypass (OAGB) $(30,563 ; 4.8 \%)[1,2]$. While the benefits of bariatric surgery are unquestionable, namely, weight loss and remission of comorbidities, surgeons must consider the risk of complications, especially because bariatric surgery is an elective procedure. The perioperative morbidity for all Clavien-Dindo complication grades after bariatric surgery is relatively low at $10.1 \%$, with treatment according to the Enhanced Recovery After Bariatric Surgery (ERABS) protocol [3]. Once complications occur, they are an issue for both the patient and the surgeon. Length of hospital stay (LOS) after bariatric surgery is $\leq 3$ days, which limits close patient monitoring postoperatively [4]. Therefore, it is particularly important to identify specific markers for early detection of complications. In centers with implemented ERAS and ERABS protocols, discharges from hospital usually occur at postoperative day (POD) 2, sometimes at POD $1[3,5]$. The goal of this study was to find early markers, even nonspecific, i.e., without highest sensitivity and specificity possible. Those markers would indicate patients, who potentially develop perioperative morbidity and who should have more attention and probably should stay longer at hospital. Inflammatory marker measurement may achieve this goal and has met similar targets in several surgical fields [6-10]. We aimed to determine the correlations between $\mathrm{C}$-reactive protein (CRP) and white blood count (WBC), both measured routinely on the first POD, and perioperative morbidity in the first 30 days after LSG, OAGB, and LRYGB.

\section{Materials and Methods}

\section{Methods}

This was a retrospective cohort study analyzing data for patients who underwent surgical treatment for morbid obesity in seven referral bariatric centers from February 2014 to March 2018. Each participating center provided specific data, which were processed and used in the overall analysis. The study was designed and performed according to the STROBE guidelines for observational studies [11]. The included patients underwent LSG, LRYGB, or OAGB, which are the most commonly performed bariatric procedures in these centers. Exclusion criteria were revision surgeries; bariatric operations other than LSG, LRYGB, or OAGB; extended surgeries during which other procedures were performed; and lack of necessary data or patients lost to follow-up. All bariatric operations in the participating centers were performed laparoscopically using comparable surgical techniques, and perioperative care was performed according to enhanced recovery after surgery protocols $($ ERAS $\AA ~[3,5,12])$, which ensured reliable data comparison. Part of our study was a review of the medical records from the databases of the participating centers. Baseline patients' characteristics were sex, age, body mass index, relevant comorbidities, and the bariatric procedure.

The primary endpoint was determining the usefulness of routinely tested inflammatory markers on POD 1, i.e., CRP and $\mathrm{WBC}$, to predict postoperative morbidity after LSG, LRYGB, and OAGB.

Patients were divided into two groups: a complicated group constituting patients with postoperative complications, and a non-complicated group constituting patients without postoperative complications. Additionally, patients' data were analyzed separately for LSG and gastric bypass (LRYGB and OAGB).

We defined postoperative morbidity as any deviation from the standard postoperative course requiring additional adequate treatment measures within 30 days of the initial procedure classified according to the Clavien-Dindo grading system [13]. LOS was defined as the period from surgery to discharge. Prolonged LOS was defined as $>4$ days because, depending on the treatment protocols in participating centers, planned hospitalization time for bariatric surgery was 3 or 4 days. Readmissions were analyzed only if they occurred in the index hospitals.

CRP and WBC were measured at each participating center on POD 1 in hospital laboratories with ISO 9001 certificates, using comparable laboratory methods. CRP was measured in blood serum using an immunonephelometric technique, and WBC was measured in full blood samples preserved with ethylenediaminetetraacetic acid, using flow cytometry.

\section{Statistical Analysis}

Statistical analyses were performed using Statistica 13.5 software (StatSoft ${ }^{\circledR}$, Tulsa, OK). Continuous values were presented as means with standard deviations or medians with interquartile ranges, as appropriate. Quantitative variables were compared using Student's $t$ test or the Mann-Whitney $U$ test, while qualitative variables were compared with the chisquared test with or without Yates' correction. Optimal cut-off points for laboratory parameters were chosen using Youden's index. Multivariate logistic regression models adjusted for relevant intergroup baseline differences were performed to 
calculate odds ratios (ORs) with $95 \%$ confidence intervals (CIs). $P$ values $\leq 0.05$ were considered statistically significant.

\section{Study Group}

The study group constituted 1400 patients from seven bariatric centers with complete data for a 30-day postoperative follow-up. Figure 1 is a patient flow chart. There were 929 women $(66 \%)$ and 471 men (34\%) in the study group, with a median age of 42 years (range, 35-51 years); 1192 patients underwent LSG (85\%), 120 underwent LRYGB (9\%), and 80 underwent OAGB $(6 \%)$.

\section{Results}

\section{Group Characteristics}

Perioperative morbidity was encountered in 69 patients (4.93\%), and morbidity rates differed significantly between LSG and bypass surgery $(p<0.001)$. Mortality was $0.29 \%$ (four patients). The general characteristics of the study groups and differences in perioperative laboratory results are presented in Table 1.

\section{Main Outcomes}

Perioperative morbidity data after LSG and bypass surgery are presented in Table 2.
Regarding the cut-off points for CRP and WBC for perioperative complications, we determined these separately for LSG and bypass surgery. We were able to determine the cutoff points for all analyzed inflammatory markers for LSG for general morbidity and morbidity of III-V Clavien-Dindo grades; however, for LRYGB and OAGB, we were able to determine a significant cut-off for only WBC on POD 1 (Table 3).

The cut-off points and other possible risk factors for perioperative morbidity after LSG were analyzed in logistic regression models and are presented in Table 4. Factors that were significant in the univariate logistic regression models were selected for multivariate analyses. CRP $>33.32 \mathrm{mg} / \mathrm{L}$ and $\mathrm{WBC}>12.15 \times 10^{3} / \mu \mathrm{L}$ on POD 1 , and longer operating time with LSG, were prognostic factors for LSG-related morbidity (Table 4).

Then, the same analyses were created for perioperative morbidity of III-V Clavien-Dindo grades after LSG. WBC on POD $1>12.2 \times 10^{3} / \mu \mathrm{L}$, determined in the ROC analysis was the only significant risk factor for perioperative morbidity of III-V Clavien-Dindo grades after LSG (Table 5).

We analyzed the cut-off points and other possible risk factors for perioperative morbidity after LRYGB and OAGB using logistic regression models, and results are presented in Table 5. In the univariate analyses, type 2 diabetes and WBC on POD 1 were significant risk factors. We found that type 2 diabetes and $\mathrm{WBC}>13.78 \times 10^{3} / \mu \mathrm{L}$ on POD 1 indicated significantly increased risk for perioperative complications after LRYGB and OAGB (Table 6).
Fig. 1 Flow chart of patients through the study

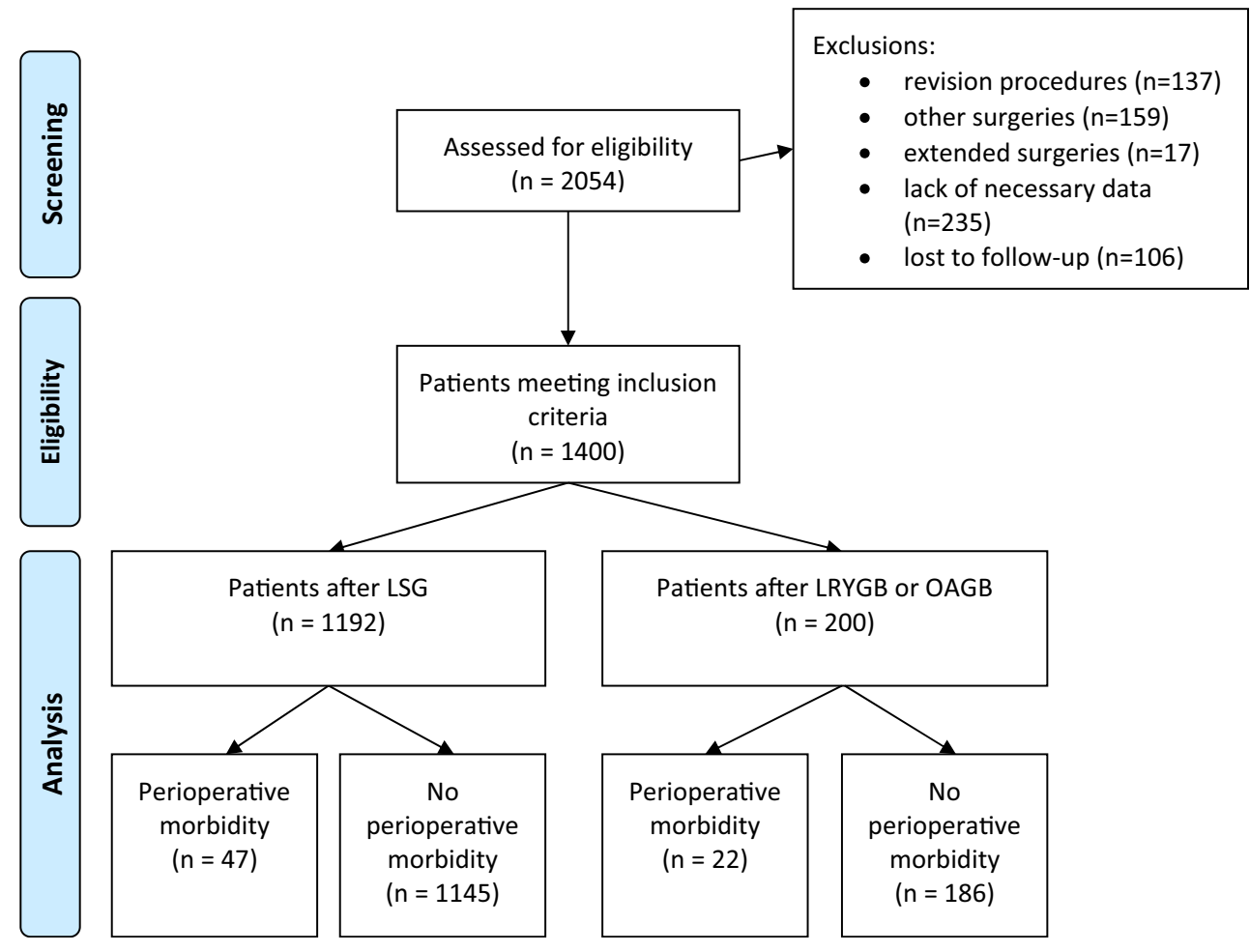


Table 1 General characteristics patients after LSG or LRYGB and OAGB

\begin{tabular}{|c|c|c|c|}
\hline & Complicated & Non-complicated & $\mathrm{p}$ value \\
\hline \multicolumn{4}{|l|}{ LSG } \\
\hline$n(\%)$ & $47(3.94 \%)$ & $1145(96.06 \%)$ & $\mathrm{n} / \mathrm{a}$ \\
\hline Males/females, $n(\%)$ & $14 / 33(30 \% / 70 \%)$ & $402 / 743(35 \% / 65 \%)$ & 0.453 \\
\hline Median age, years (IQR) & $40(35-49)$ & $41(34-50)$ & 0.944 \\
\hline Median BMI, kg/m² (IQR) & $44.46(39.31-49.84)$ & $43.59(40.27-48)$ & 0.435 \\
\hline Diabetes mellitus type $2, n(\%)$ & $13(27.66 \%)$ & $251(21.96 \%)$ & 0.357 \\
\hline Arterial hypertension, $n(\%)$ & $28(59.57 \%)$ & $575(50.22 \%)$ & 0.211 \\
\hline Obstructive sleep apnea, $n(\%)$ & $7(14.89 \%)$ & $147(12.84 \%)$ & 0.637 \\
\hline Median operative time, $\min (\mathrm{IQR})$ & $75(60-105)$ & $70(55.5-90)$ & 0.113 \\
\hline Median LOS, days (IQR) & $5(4-7)$ & $3(3-4)$ & $<0.001$ \\
\hline Readmissions, $n(\%)$ & $12(25.53 \%)$ & $9(0.79 \%)$ & $<0.001$ \\
\hline Median CRP on POD 1, mg/L (IQR) & $28.77(15.1-48.8)$ & $18.84(11.6-29.6)$ & 0.002 \\
\hline Median WBC on POD $1, \times 10^{3} / \mu \mathrm{L}(\mathrm{IQR})$ & $12.42(10.7-16.83)$ & $11.16(9.25-13.21)$ & 0.002 \\
\hline \multicolumn{4}{|l|}{ Bypasses } \\
\hline$n(\%)$ & $22(10.58 \%)$ & $186(89.42 \%)$ & $\mathrm{n} / \mathrm{a}$ \\
\hline Males/females, $n(\%)$ & $9 / 13(41 \% / 59 \%)$ & $46 / 140(25 \% / 75 \%)$ & 0.088 \\
\hline Median age, years (IQR) & $44(40-50)$ & $46(39-54)$ & 0.188 \\
\hline Median BMI, kg/m² (IQR) & $42.05(37-46.60)$ & $42.05(38.30-46.66)$ & 0.857 \\
\hline Diabetes mellitus type $2, n(\%)$ & $11(50.0 \%)$ & $42(22.58 \%)$ & 0.022 \\
\hline Arterial hypertension, $n(\%)$ & $14(63.64 \%)$ & $112(60.22 \%)$ & 0.756 \\
\hline Obstructive sleep apnea, $n(\%)$ & $4(18.18 \%)$ & $34(18.28 \%)$ & 0.965 \\
\hline Median operative time, $\min (\mathrm{IQR})$ & $95(65-150)$ & $95(65-150)$ & 0.810 \\
\hline Median LOS, days (IQR) & $4(4-8)$ & $3(2-4)$ & 0.003 \\
\hline Readmissions, $n(\%)$ & $7(31.82 \%)$ & $5(2.69 \%)$ & $<0.001$ \\
\hline Median CRP on POD 1, mg/L (IQR) & $13.51(6.7-27.76)$ & $18.68(10-41.1)$ & 0.316 \\
\hline Median WBC on POD $1, \times 10^{3} / \mu \mathrm{L}$ (IQR) & $15.69(13.88-16.78)$ & $11.28(9.47-13.23)$ & $<0.001$ \\
\hline
\end{tabular}

$B M I$, body mass index; $I Q R$, interquartile range; $L O S$, length of hospital stay; $C R P$, C-reactive protein; $W B C$, white blood count; $P O D$, postoperative day
In case of perioperative morbidity of III-V Clavien-Dindo grades, the only significant parameter in univariate logistic regression models was $\mathrm{WBC}$ on $\mathrm{POD} 1>13.78 \times 103 / \mu \mathrm{L}$ (Table 7).

\section{Discussion}

Bariatric procedures are now considered standard in general surgery in Poland. Moreover, these procedures are performed in low-volume centers and private facilities, often as a singleday admission or as medical tourism. Therefore, identifying early markers predicting perioperative morbidity is needed and relevant, as the patients are usually discharged from hospital at POD 2. We found similar research and study protocols for early markers of morbidity in other fields of general surgery, particularly in colorectal surgery, but we found no studies with a large patient cohort undergoing bariatric surgery analyzing the usefulness of CRP and WBC to predict the risk of perioperative complications, as in our study.
We evaluated patients undergoing surgery for morbid obesity in accordance with the ERAS® protocols [12]. Our patient groups were similar regarding their baseline characteristics, and our results showed that CRP and WBC values differed between patients who developed complications and those who did not. Multivariate analysis and ROC analysis showed that CRP $>33.32 \mathrm{mg} / \mathrm{L}$ on POD 1 was associated with a three times greater risk of developing complications, and that $\mathrm{WBC}>12.15 \times 10^{3} / \mu \mathrm{L}$ on POD 1 was associated with a two times greater risk in patients undergoing LSG. The only inflammatory marker that was significantly associated with a higher risk of morbidity after gastric bypass was $\mathrm{WBC}>13.78 \times 10^{3} / \mu \mathrm{L}$ on POD 1 , which increased the risk by 13 times. In case of perioperative morbidity of III-V Clavien-Dindo grades, WBC on POD 1 remained indicative risk factor for perioperative morbidity after LSG, as well as bypasses.

Albanopoulos et al., in their study evaluating LSG, showed that WBC and CRP were correlated with leakage or abscess on PODs 3, 5, 7, 9, and 11 [6]. However, the main 
Table 2 Perioperative morbidity sorted out regarding ClavienDindo classification of surgical complications

\begin{tabular}{|c|c|c|c|}
\hline $\mathrm{C}-\mathrm{D}$ & Perioperative & LSG & LRYGB and OAGB \\
\hline \multirow[t]{4}{*}{$\mathrm{V}$} & Urosepsis & 1 & 0 \\
\hline & GI leak & 1 & 0 \\
\hline & Intra-abdominal hernia, GI leak & 0 & 1 \\
\hline & Acute pancreatitis, GI leak, peritonitis & 1 & 0 \\
\hline $\mathrm{IVb}$ & Acute respiratory failure & 1 & 0 \\
\hline IVa & Heart failure & 1 & 0 \\
\hline \multirow[t]{4}{*}{ IIIb } & Subphrenic abscess & 2 & 1 \\
\hline & GI leak & 5 & 3 \\
\hline & Postoperative bleeding (reoperation) & 11 & 3 \\
\hline & GI stricture & 0 & 2 \\
\hline \multirow[t]{5}{*}{ IIIa } & GI stricture & 1 & 1 \\
\hline & Postoperative bleeding (treated endoscopically) & 4 & 7 \\
\hline & Surgical site infection & 3 & 1 \\
\hline & Intraperitoneal fluid collection & 1 & 0 \\
\hline & Subphrenic hematoma, acute pancreatitis & 1 & 0 \\
\hline \multirow[t]{5}{*}{ II } & Postoperative bleeding (treated with blood transfusions) & 2 & 3 \\
\hline & Renal insufficiency & 1 & 0 \\
\hline & Clostridium difficile infection & 1 & 0 \\
\hline & Superior mesenteric vein and/or portal vein thrombosis & 2 & 0 \\
\hline & Pneumonia & 2 & 0 \\
\hline I & Vomiting/dehydration & 6 & 1 \\
\hline Total & & $47(3.94 \%)$ & $22(10.58 \%)$ \\
\hline
\end{tabular}

disadvantage of the study was the low number of patients with complications, which rendered the conclusions difficult to interpret. Mickevicius et al. presented results after LRYGB and showed that CRP differed significantly on POD 1 between patients with and without postoperative 30-day morbidity. This finding differs from our results regarding LRYGB, which indicated that differences in CRP levels were non-significant [14]. Villard et al. identified CRP as a specific marker, however, with very low sensitivity [15]. In their study, the authors identified CRP of $50 \mathrm{mg} / \mathrm{L}$ as a predictor of complications. In contrast, our multivariate analysis predicted complications when CRP was $>33.32 \mathrm{mg} / \mathrm{L}$. The main advantage in our analysis is that our regression models included additional factors. Williams et al. reported even higher CRP levels at > $90 \mathrm{mg} / \mathrm{L}$ on POD 1 in patients with complications and $75 \mathrm{mg} / \mathrm{L}$ in patients without complications. Warschkow et al. suggested that CRP should be routinely measured on POD 2 after LRYGB to exclude complications, and leaks in particular [16]. The authors concluded that radiological imaging examinations for intestinal leaks could be restricted to patients with

Table 3 Results of ROC analyses of CRP on POD $1(\mathrm{mg} / \mathrm{L})$ and WBC on POD $1\left(\times 10^{3} / \mu \mathrm{L}\right)$ for LSG and bypasses

\begin{tabular}{|c|c|c|c|c|c|c|}
\hline & & Cut-off point & AUC $(95 \% \mathrm{CI})$ & $\mathrm{p}$ value & Sensitivity (\%) & Specificity (\%) \\
\hline \multicolumn{7}{|l|}{ LSG } \\
\hline \multirow[t]{2}{*}{ Morbidity } & CRP & 33.32 & $0.66(0.56-0.76)$ & 0.002 & 47.1 & 80.2 \\
\hline & WBC & 12.15 & $0.65(0.56-0.74)$ & 0.001 & 48.7 & 66.9 \\
\hline \multirow[t]{2}{*}{ Morbidity of III-V Clavien-Dindo } & CRP & 35.00 & $0.59(0.46-0.72)$ & 0.048 & 36.4 & 81.5 \\
\hline & WBC & 12.20 & $0.69(0.58-0.579)$ & 0.001 & 66.7 & 63.3 \\
\hline \multicolumn{7}{|l|}{ Bypasses } \\
\hline \multirow[t]{2}{*}{ Morbidity } & CRP & 82.75 & $0.42(0.26-0.58)$ & 0.332 & $\mathrm{n} / \mathrm{a}$ & $\mathrm{n} / \mathrm{a}$ \\
\hline & $\mathrm{WBC}$ & 13.78 & $0.8(0.69-0.90)$ & $<0.001$ & 81.8 & 79.6 \\
\hline \multirow[t]{2}{*}{ Morbidity of III-V Clavien-Dindo } & CRP & 92.7 & $0.47(0.25-0.69)$ & 0.778 & $\mathrm{n} / \mathrm{a}$ & $\mathrm{n} / \mathrm{a}$ \\
\hline & WBC & 13.78 & $0.79(0.66-0.92)$ & $<0.001$ & 85.7 & 77.3 \\
\hline
\end{tabular}


Table 4 Logistic regression models of risk factors for perioperative morbidity after LSG

\begin{tabular}{lccc}
\hline & OR & $95 \%$ CI & $p$ value \\
\hline Univariate & & & \\
Females & 1.13 & $0.82-1.55$ & 0.454 \\
Age, every year & 1.00 & $0.97-1.03$ & 0.975 \\
BMI, every kg/m ${ }^{2}$ & 1.02 & $0.98-1.07$ & 0.268 \\
Diabetes mellitus type 2 & 1.17 & $0.84-1.62$ & 0.358 \\
Arterial hypertension & 1.21 & $0.90-1.63$ & 0.213 \\
Obstructive sleep apnea & 1.10 & $0.73-1.67$ & 0.638 \\
Operative time, every min & 1.01 & $1.00-1.02$ & 0.034 \\
CRP on POD $1>33.32 \mathrm{mg} / \mathrm{L}$ & 3.19 & $1.57-6.49$ & 0.001 \\
WBC on POD $1>12.15 \times 10^{3} / \mu \mathrm{L}$ & 2.20 & $1.15-4.21$ & 0.018 \\
Multivariate & & & \\
Operative time, every min & 1.02 & $1.00-1.03$ & 0.016 \\
CRP on POD $1>33.32 \mathrm{mg} / \mathrm{L}$ & 2.27 & $1.02-5.02$ & 0.043 \\
WBC on POD $1>12.15 \times 10^{3} / \mu \mathrm{L}$ & 3.34 & $1.54-7.25$ & 0.002 \\
\hline
\end{tabular}

$O R$, odds ratio; $95 \% C I, 95 \%$ confidence interval; $B M I$, body mass index; $C R P, \mathrm{C}$-reactive protein; $W B C$, white blood count; $P O D$, postoperative day

serum CRP $>229 \mathrm{mg} / \mathrm{L}$ on POD 2. Accordingly, it is difficult to determine the exact cut-off point for CRP concentration to predict complications.

WBC count in a study by Mickevicius et al. did not differ between the groups. Similar results were presented by RuizTovar et al. [17], and a study by Da Silva et al. evaluated the correlation between neutrophil-to-lymphocyte ratio (NLR) on POD 1 as a predictive factor for perioperative complications [18]. Da Silva et al.'s study was a retrospective cohort study of 737 patients who underwent predominantly LRYGB, but also

Table 5 Logistic regression models of risk factors for perioperative morbidity of III-V Clavien-Dindo after LSG

\begin{tabular}{lccc}
\hline & OR & $95 \%$ CI & $p$ value \\
\hline Univariate & & & \\
Females & 1.07 & $0.49-2.36$ & 0.859 \\
Age, every year & 0.99 & $0.96-1.03$ & 0.714 \\
BMI, every kg/m ${ }^{2}$ & 1.02 & $0.97-1.07$ & 0.449 \\
Diabetes mellitus type 2 & 1.07 & $0.45-2.54$ & 0.879 \\
Arterial hypertension & 1.12 & $0.54-2.32$ & 0.765 \\
Obstructive sleep apnea & 0.64 & $0.22-2.49$ & 0.629 \\
Operative time, every $\mathrm{min}$ & 1.01 & $1.00-1.02$ & 0.080 \\
CRP on POD $1>35 \mathrm{mg} / \mathrm{L}$ & 2.06 & $0.82-5.18$ & 0.126 \\
WBC on POD $1>12.2 \times 10^{3} / \mu \mathrm{L}$ & 2.87 & $1.24-6.65$ & 0.014 \\
\hline
\end{tabular}

$O R$, odds ratio; $95 \% C I, 95 \%$ confidence interval; $B M I$, body mass index; $C R P$, C-reactive protein; $W B C$, white blood count; $P O D$, postoperative day
Table 6 Logistic regression models of risk factors for postoperative morbidity after bypasses

\begin{tabular}{lrrr}
\hline & OR & $95 \%$ CI & $p$ value \\
\hline Univariate & & & \\
Females & 0.48 & $0.19-1.18$ & 0.110 \\
Age & 0.97 & $0.93-1.02$ & 0.190 \\
BMI & 1.00 & $0.94-1.07$ & 0.913 \\
Diabetes mellitus type 2 & 3.23 & $1.12-9.09$ & 0.029 \\
Arterial hypertension & 1.16 & $0.46-2.89$ & 0.756 \\
Obstructive sleep apnea & 1.03 & $0.28-3.86$ & 0.965 \\
Operative time & 1.00 & $0.99-1.01$ & 0.855 \\
CRP on POD 1, every mg $/ \mathrm{L}$ & 1.00 & $0.98-1.02$ & 0.946 \\
WBC on POD 1 > 13.78 $\times 10^{3} / \mu \mathrm{L}$ & 13.24 & $4.59-38.18$ & $<0.001$ \\
Multivariate & & & \\
Diabetes mellitus type 2 & 3.13 & $1.04-9.48$ & 0.043 \\
WBC on POD $1>13.78 \times 10^{3} / \mu \mathrm{L}$ & 13.14 & $4.50-38.41$ & $<0.001$ \\
\hline
\end{tabular}

$O R$, odds ratio; $95 \% C I, 95 \%$ confidence interval; $B M I$, body mass index; $C R P$, C-reactive protein; $W B C$, white blood count; $P O D$, postoperative day

LSG. The authors reported that NLR $\geq 10$ on POD 1 was associated with the following 30-day clinical outcomes: prolonged hospital stay ( $>2$ days); higher incidence of overall complications and major complications as well as higher readmission rate; and higher reoperation rate. On multivariable analysis, elevated NLR retained its predictive value for all outcome variables, except for readmissions.

Interestingly, prolonged operative time was a significant predictor for perioperative morbidity in patients undergoing LSG, in our study. We believe that this finding was not a result of longer operation times, but, rather, was secondary to

Table 7 Logistic regression models of risk factors for perioperative morbidity of III-V Clavien-Dindo after bypasses

\begin{tabular}{lccr}
\hline & OR & $95 \%$ CI & $p$ value \\
\hline Univariate & & & \\
Females & 0.63 & $0.20-1.97$ & 0.419 \\
Age, every year & 0.96 & $0.91-1.02$ & 0.165 \\
BMI, every kg/m ${ }^{2}$ & 1.01 & $0.64-1.09$ & 0.788 \\
Diabetes mellitus type 2 & 0.45 & $0.14-1.50$ & 0.193 \\
Arterial hypertension & 1.68 & $0.51-5.59$ & 0.394 \\
Obstructive sleep apnea & 0.43 & $0.05-3.52$ & 0.425 \\
Operative time, every min & 1.00 & $0.99-1.01$ & 0.635 \\
WBC on POD $1>13.78 \times 10^{3} / \mu \mathrm{L}$ & 12.5 & $3.31-47.16$ & $<0.001$ \\
Multivariate & & & \\
\hline
\end{tabular}

$O R$, odds ratio; $95 \% C I, 95 \%$ confidence interval; $B M I$, body mass index; $C R P$, C-reactive protein; $W B C$, white blood count; $P O D$, postoperative day 
technical difficulties or intraoperative adverse events, which we did not analyze in this study [4, 19]. Major et al. evaluated morbidity after LSG and reported that prolonged operation and increased number of stapler firings were associated with a higher risk of perioperative complications, but interestingly, intraoperative adverse events were not directly associated with an increased risk of perioperative morbidity. In another study by Major et al., decreased oral fluid intake, the need for increased intravenous fluids on the day of surgery, and longer distance from the patient's habitual residence to the bariatric center were potential risk factors for prolonged hospital stay. Intraoperative adverse events also increased the risk for hospital readmission [4, 19].

In patients undergoing colorectal surgery, measuring inflammatory markers is now almost a routine. However, colorectal surgery has different specificity and higher rates for postoperative morbidity, and leaks are more prevalent, which determines different cut-off points. In a meta-analysis by Cousin et al. of the accuracy of CRP on POD 3 to diagnose intra-abdominal infection after elective colorectal surgery, the cut-offs varied in the studies, from 130 to $190 \mathrm{mg} / \mathrm{L}$ [20]. CRP and procalcitonin were significantly higher much earlier than when patients became symptomatic, at between POD 7 and POD 9 [21-23]. This finding is crucial to understand why we performed a similar study in bariatric surgery. Significantly higher CRP levels were found after colorectal surgery as early as POD 1 in patients who later developed intra-abdominal infection, which raises doubts regarding opinions that anastomotic leakage occurs near POD 7 [20, 22, 24]. A stronger inflammatory response may be both the consequence and the cause of intra-abdominal infection (impaired healing or reflecting ongoing tissue hypoxia leading to anastomotic leakage) $[21,25]$.

The main advantage of our study is the high patient numbers, which makes our conclusions more reliable. We found no ERAS $®$ guidelines indicating laboratory measurements predicting postoperative morbidity. It would be useful to have cut-off points for laboratory measurements of inflammatory markers to select patients requiring longer postoperative stays, for early detection and prevention of postoperative morbidity. Short hospital stays are necessary to maintain a high bariatric surgical volume; therefore, having laboratory criteria for early and safe discharge from hospital would be highly beneficial [26].

\section{Limitations}

This was a non-randomized analysis, and our groups were demographically heterogeneous and differed regarding the preoperative factors. Furthermore, because the data were collected separately from seven bariatric centers using different electronic systems, some necessary information was lacking in our initial population, which caused exclusions. After excluding incomplete records and repetitions in the collective database, we obtained our final number of patients, which added selection bias. Additionally, we did not record postoperative events, such as readmissions occurring outside the indexed hospitals. Patients during hospital discharge were informed to report to index hospital in case of any emergencies. In case of patients that live in a long distance they could refer to local hospitals.

\section{Conclusion}

$\mathrm{CRP}>33.32 \mathrm{mg} / \mathrm{L}$ and $\mathrm{WBC}>12.15 \times 10^{3} / \mu \mathrm{L}$ on POD 1 were associated with a greater risk of developing complications in patients undergoing LSG. WBC $>13.78 \times 10^{3} / \mu \mathrm{L}$ was associated with a higher risk of morbidity in patients after bypass surgery. Even slightly elevated CRP and WBC on POD 1 should alert surgeons to the potential risk of perioperative morbidity.

\section{Compliance with Ethical Standards}

Conflicts of Interest The authors declare that they have no conflict of interest.

Ethical Approval All procedures performed in studies involving human participants were in accordance with the ethical standards of the institutional and/or national research committee and with the 1964 Helsinki declaration and its later amendments or comparable ethical standards.

Open Access This article is licensed under a Creative Commons Attribution 4.0 International License, which permits use, sharing, adaptation, distribution and reproduction in any medium or format, as long as you give appropriate credit to the original author(s) and the source, provide a link to the Creative Commons licence, and indicate if changes were made. The images or other third party material in this article are included in the article's Creative Commons licence, unless indicated otherwise in a credit line to the material. If material is not included in the article's Creative Commons licence and your intended use is not permitted by statutory regulation or exceeds the permitted use, you will need to obtain permission directly from the copyright holder. To view a copy of this licence, visit http://creativecommons.org/licenses/by/4.0/.

\section{References}

1. Angrisani L, Santonicola A, Iovino P, et al. IFSO Worldwide Survey 2016: primary, endoluminal, and revisional procedures. Obes Surg. 2018;28:3783-94. United States

2. Angrisani L, Santonicola A, Iovino P, et al. Bariatric surgery and endoluminal procedures: IFSO Worldwide Survey 2014. Obes Surg. 2017;27:2279-89. United States

3. Małczak P, Pisarska M, Piotr M, et al. Enhanced recovery after bariatric surgery: systematic review and meta-analysis. Obes Surg. 2017;27:226-35. United States

4. Major P, Wysocki M, Torbicz G, et al. Risk factors for prolonged length of hospital stay and readmissions after laparoscopic sleeve gastrectomy and laparoscopic Roux-en-Y gastric bypass. Obes 
Surg. 2018;28(2):323-332. https://doi.org/10.1007/s11695-0172844-x.

5. Proczko M, Kaska L, Twardowski P, et al. Implementing enhanced recovery after bariatric surgery protocol: a retrospective study. $\mathrm{J}$ Anesth. 2016;30:170-3.

6. Albanopoulos K, Alevizos L, Natoudi M, et al. C-reactive protein, white blood cells, and neutrophils as early predictors of postoperative complications in patients undergoing laparoscopic sleeve gastrectomy. Surg Endosc. 2013;27:864-71.

7. Dib F, Parenti LR, Boutten A, et al. Diagnostic performance of Creactive protein in detecting post-operative infectious complications after laparoscopic sleeve gastrectomy. Obes Surg. 2017;27(12): 3124-32. https://doi.org/10.1007/s11695-017-2744-0.

8. Park J, Chung K, Song J, et al. The C-reactive protein/albumin ratio as a predictor of mortality in critically ill patients. J Clin Med. 2018;7(10):333. https://doi.org/10.3390/jcm7100333.

9. Wu J-M, Yang H-T, Ho T-W, et al. Association between interleukin6 levels and perioperative fatigue in gastric adenocarcinoma patients. J Clin Med. 2019;8(4):543. https://doi.org/10.3390/ jem8040543.

10. Zawadzki M, Krzystek-Korpacka M, Gamian A, et al. Serum cytokines in early prediction of anastomotic leakage following low anterior resection. Videosurgery Other Miniinvasive Tech. 2018;13: 33-43. https://doi.org/10.5114/wiitm.2018.72785. [Internet]

11. von Elm E, Altman DG, Egger M, et al. The Strengthening the reporting of observational studies in epidemiology (STROBE) statement: guidelines for reporting observational studies. Int J Surg. 2014;12(12):1495-9. https://doi.org/10.1016/j.ijsu.2014.07. 013 .

12. Thorell A, MacCormick AD, Awad S, et al. Guidelines for perioperative care in bariatric surgery: enhanced recovery after surgery (ERAS) society recommendations. World J Surg. 2016;40:206583.

13. Dindo D, Demartines N, Clavien PA. Classification of surgical complications: a new proposal with evaluation in a cohort of 6336 patients and results of a survey. Ann Surg. 2004;240:205-13.

14. Mickevicius A, Sufi P, Heath D. Factors predicting the occurrence of a gastrojejunal anastomosis leak following gastric bypass. Wideochir Inne Tech Maloinwazyjne. 2014 Sep;9(3):436-40. https://doi.org/10.5114/wiitm.2014.44142.

15. Villard MA, Helm MC, Kindel TL, et al. C-Reactive protein as a predictor of post-operative complications in bariatric surgery patients. Surg Endosc. 2019;33:2479-84. Germany

16. Warschkow R, Tarantino I, Folie P, et al. C-Reactive protein 2 days after laparoscopic gastric bypass surgery reliably indicates leaks and moderately predicts morbidity. J Gastrointest Surg. 2012;16: $1128-35$.
17. Ruiz-Tovar J, Muñoz JL, Gonzalez J, et al. C-reactive protein, fibrinogen, and procalcitonin levels as early markers of staple line leak after laparoscopic sleeve gastrectomy in morbidly obese patients within an enhanced recovery after surgery (ERAS) program. Surg Endosc. 2017;31:5283-8.

18. Da Silva M, Cleghorn MC, Elnahas A, et al. Postoperative day one neutrophil-to-lymphocyte ratio as a predictor of 30-day outcomes in bariatric surgery patients. Surg Endosc. 2017;31(6):2645-50. https://doi.org/10.1007/s00464-016-5278-y.

19. Major P, Wysocki M, Dworak J, et al. Analysis of laparoscopic sleeve gastrectomy learning curve and its influence on procedure safety and perioperative complications. Obes Surg. 2018;28(6): 1672-80. https://doi.org/10.1007/s11695-017-3075-x.

20. Cousin F, Ortega-Deballon P, Bourredjem A, et al. Diagnostic accuracy of procalcitonin and C-reactive protein for the early diagnosis of intra-abdominal infection after elective colorectal surgery: a meta-analysis. Ann Surg. 2016;264:252-6.

21. Warschkow R, Beutner U, Steffen T, et al. Safe and early discharge after colorectal surgery due to C-reactive protein: a diagnostic metaanalysis of 1832 patients. Ann Surg. 2012;256(2):245-50. https:// doi.org/10.1097/SLA.0b013e31825b60f0.

22. Facy O, Paquette B, Orry D, et al. Diagnostic accuracy of inflammatory markers as early predictors of infection after elective colorectal surgery. Results from the IMACORS study. Ann Surg. 2016;263(5):961-6. https://doi.org/10.1097/S LA. 0000000000001303 .

23. Ortega-Deballon P, Radais F, Facy O, et al. C-reactive protein is an early predictor of septic complications after elective colorectal surgery. World J Surg. 2010;34:808-14.

24. Woeste G, Müller C, Bechstein WO, et al. Increased serum levels of C-reactive protein precede anastomotic leakage in colorectal surgery. World J Surg. 2010;34(1):140-6. https://doi.org/10.1007/ s00268-009-0304-z.

25. Wierdak M, Pisarska M, Kuśnierz-Cabala B, et al. Use of inflammatory markers in the early detection of infectious complications after laparoscopic colorectal cancer surgery with the ERAS protocol. Wideochir Inne Tech Maloinwazyjne. 2018;13(3):315-25. https://doi.org/10.5114/wiitm.2018.75846.

26. van Mil SR, Duinhouwer LE, Mannaerts GHH, et al. The standardized postoperative checklist for bariatric surgery; a tool for safe early discharge? Obes Surg. 2017;27(12):3102-09. https://doi.org/ 10.1007/s11695-017-2746-y.

Publisher's Note Springer Nature remains neutral with regard to jurisdictional claims in published maps and institutional affiliations. 


\section{Affiliations}

Michał Wysocki ${ }^{1} \cdot$ Piotr Małczak ${ }^{1} \cdot$ Mateusz Wierdak $^{1} \cdot$ Maciej Walędziak $^{2} \cdot$ Hady Razak Hady $^{3} \cdot$ Inna Diemieszczyk $^{3}$. Monika Proczko-Stepaniak ${ }^{4}$ Michał Szymański ${ }^{4}$. Natalia Dowgiałło-Wnukiewicz ${ }^{5}$. Jacek Szeliga ${ }^{6}$.

Michał Pędziwiatr ${ }^{1} \cdot$ Piotr Major $^{1}$ (i)

1 2nd Department of General Surgery, Jagiellonian University Medical College, Jakubowskiego 2 St., 30-688 Cracow, Poland

2 Department of General, Oncological, Metabolic and Thoracic Surgery, Military Institute of Medicine, Warsaw, Poland

3 First Department of General and Endocrine Surgery, Medical University of Bialystok, Bialystok, Poland
4

Department of General, Endocrine and Transplant Surgery, Medical University of Gdańsk, Gdańsk, Poland

5 Department of General, Minimally Invasive and Elderly Surgery, University of Warmia and Mazury, Olsztyn, Poland

6 Department of General, Gastroenterological, and Oncological Surgery, Collegium Medicum Nicolaus Copernicus University, Torun, Poland 